\title{
Sun-sized Water Vapor Masers in Cepheus A
}

\author{
A. M. Sobolev ${ }^{1}$, J. M. Moran ${ }^{2}$, M. D. Gray ${ }^{3}$, A. Alakoz ${ }^{4}$, H. Imai ${ }^{5}$, W. A. Baan ${ }^{6}$, A. M. Tolmachev ${ }^{4}$, \\ V. A. Samodurov ${ }^{4,7}$, and D. A. Ladeyshchikov ${ }^{1}$ \\ ${ }^{1}$ Ural Federal University, Ekaterinburg, Russia \\ ${ }^{2}$ Harvard-Smithsonian Center for Astrophysics, 60 Garden Street, Cambridge, MA 02138, USA; jmoran@cfa.harvard.edu \\ ${ }^{3}$ Jodrell Bank Centre for Astrophysics, School of Physics and Astronomy, Alan Turing Building, University of Manchester, M13 9PL, UK \\ ${ }^{4}$ Astro Space Center of the Lebedev Physical Institute, Moscow, Russia \\ ${ }^{5}$ Science and Engineering Area of the Research and Education Assembly, Kagoshima University, 1-21-35 Korimoto, Kagoshima 890-0065, Japan \\ ${ }^{6}$ ASTRON-Netherlands Foundation for Research in Astronomy, Dwingeloo, The Netherlands \\ ${ }^{7}$ National Research University, Higher School of Economics, Moscow, Russia \\ Received 2017 November 21; revised 2018 February 15; accepted 2018 February 16; published 2018 March 26
}

\begin{abstract}
We present the first VLBI observations of a Galactic water maser (in Cepheus A) made with a very long baseline interferometric array involving the RadioAstron Earth-orbiting satellite station as one of its elements. We detected two distinct components at -16.9 and $0.6 \mathrm{~km} \mathrm{~s}^{-1}$ with a fringe spacing of $66 \mu$ as. In total power, the $0.6 \mathrm{~km} \mathrm{~s}^{-1}$ component appears to be a single Gaussian component of strength $580 \mathrm{Jy}$ and width of $0.7 \mathrm{~km} \mathrm{~s}^{-1}$. Single-telescope monitoring showed that its lifetime was only eight months. The absence of a Zeeman pattern implies the longitudinal magnetic field component is weaker than $120 \mathrm{mG}$. The space-Earth cross power spectrum shows two unresolved components smaller than $15 \mu$ as, corresponding to a linear scale of $1.6 \times 10^{11} \mathrm{~cm}$, about the diameter of the Sun, for a distance of $700 \mathrm{pc}$, separated by $0.54 \mathrm{~km} \mathrm{~s}^{-1}$ in velocity and by $160 \pm 35 \mu \mathrm{as}$ in angle. This is the smallest angular structure ever observed in a Galactic maser. The brightness temperatures are greater than $2 \times 10^{14} \mathrm{~K}$, and the line widths are $0.5 \mathrm{~km} \mathrm{~s}^{-1}$. Most of the flux (about $87 \%$ ) is contained in a halo of angular size of $400 \pm 150 \mu$ as. This structure is associated with the compact $\mathrm{H}$ II region HW3diii. We have probably picked up the most prominent peaks in the angular size range of our interferometer. We discuss three dynamical models: (1) Keplerian motion around a central object, (2) two chance overlapping clouds, and (3) vortices caused by flow around an obstacle (i.e., von Kármán vortex street) with a Strouhal number of about 0.3 .
\end{abstract}

Key words: ISM: individual objects (Cepheus A) - ISM: magnetic fields - masers - stars: formation - techniques: interferometric

\section{Introduction}

Cepheus $\mathrm{A}$ is a region of massive star formation within our Galaxy. Its radio continuum image consists of about 16 compact thermal cores, many of which are associated with embedded heating sources in the form of newly formed $\mathrm{O}$ and B stars. These sources were first identified by Hughes \& Wouterloot (1984) and are numbered with the prefix HW. The distance to the complex has been determined to be $700 \pm 40 \mathrm{pc}$ both by VLBI parallax measurement from the continuum emission from HW9 (Dzib et al. 2011) and methanol masers associated with HW2 (Moscadelli et al. 2009). HW2 is the dominant energy source in the complex. Its continuum emission arises from an elongated structure (see Figure 1), which has been identified as a thermal jet with an outflow velocity of $480 \mathrm{~km} \mathrm{~s}^{-1}$ (Curiel et al. 2006). Another structure, perpendicular to the jet, is a disk of dust and molecular gas (Patel et al. 2005). A system of water masers is associated with this disk, whose components are spread over an area of about 0 ". 5 in extent (Torrelles et al. 1998). Another important source is HW3, which lies about $3^{\prime \prime}$ south of HW2. The radio continuum emission shows four distinct cores, all probably associated with newly formed B stars (Hughes et al. 1995). Most of the water masers associated with HW3d define a highly collimated outflow centered on HW3dii (Chibueze et al. 2012). Of particular interest to this study is the source HW3diii, which lies about 0 ". 5 east of HW3dii. The morphology of the HW2 and HW3 regions is shown in
Figure 1. For a general discussion of the physics of cosmic masers, see Gray (2012).

We present in this paper our measurements of the maser emission from Cepheus A made with an unprecedented resolution (at the time of observations) of $66 \mu$ as on a baseline of 3.3 Earth diameters (ED). These are among the earliest results from a VLBI experiment that incorporate the RadioAstron satellite radio telescope (SRT). More recently, observations with baselines up to $10 \mathrm{ED}$ on other galactic masers and up to $26.7 \mathrm{ED}$ on extragalactic masers have been presented in conference proceedings (Baan et al. 2018; Shakhvorostova et al. 2018; Sobolev et al. 2018). The only other reported detection of an $\mathrm{H}_{2} \mathrm{O}$ maser with a space VLBI experiment was of the very bright maser in the Orion-KL region, but with projected baseline shorter than an ED (Kobayashi et al. 2000).

The properties of the SRT, which was launched in 2011, are described by Kardashev et al. (2013) and the RadioAstron User Handbook (2018). The SRT operates at frequencies of 22, 5, 1.6 , and $0.3 \mathrm{GHz}$. The receiving element is a $10 \mathrm{~m}$ parabolic dish, whose aperture efficiency is about $10 \%$ at $22 \mathrm{GHz}$. The local oscillator phase is controlled by an onboard hydrogen maser. There are four baseband channels: two subbands of $16 \mathrm{MHz}$ in each sense of circular polarization. These signal streams were digitally sampled with one-bit quantization, transmitted to Earth, and recorded for later processing at the VLBI center in Moscow. 


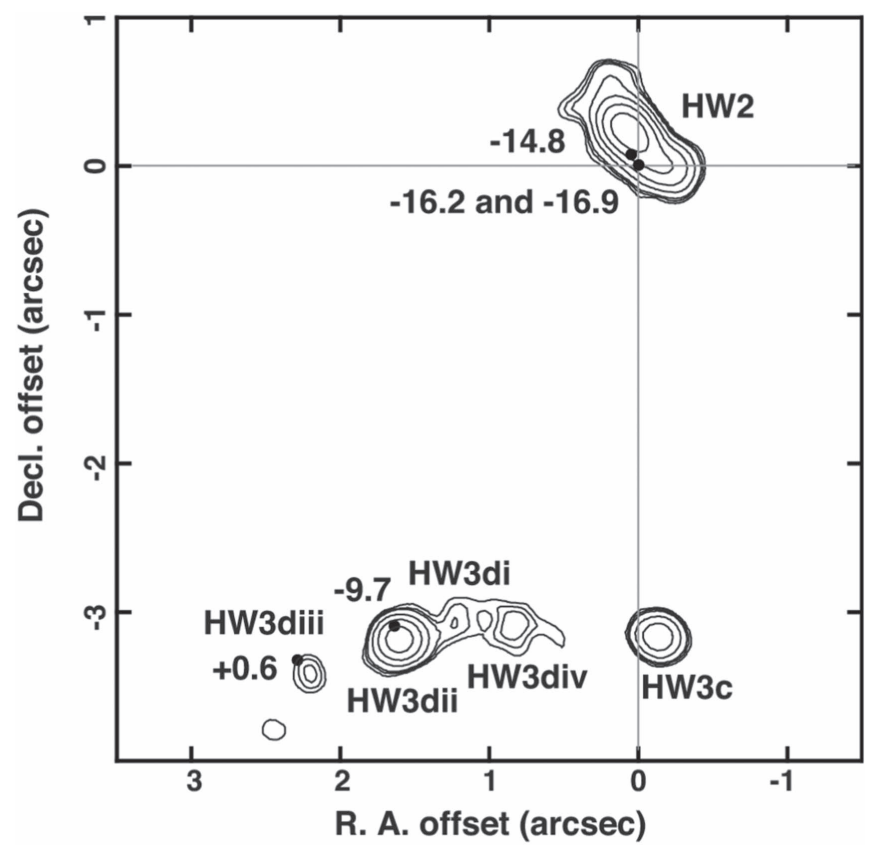

Figure 1. The central part of the star-forming region Cepheus A. The contours show the extent of the continuum components taken from the $1.3 \mathrm{~cm}$ VLA image (adapted from Torrelles et al. 1998). The nomenclature is based on the original identification of about 16 continuum radio sources marking the sites of newly formed massive stars by Hughes \& Wouterloot (1984). The dots mark the positions of masers (labeled by their velocities) whose positions were found by analysis of the relative fringe rates derived from these observations. The coordinate origin is the center of HW2/R4: R.A. $=22^{\mathrm{h}} 56^{\mathrm{m}} 17^{\mathrm{s}} .977$, decl. $=62^{\mathrm{d}} 01^{\prime} 49^{\prime \prime} 38$ (2000). The relative alignment of the masers and continuum is accurate to about \pm 0 !" 2 (see the text). At a distance of $700 \mathrm{pc}$, $1^{\prime \prime}$ corresponds to $1.05 \times 10^{16} \mathrm{~cm}$.

\section{Observations}

The observations were made in a single 40-minute period from 12:00 UT to 12:40 UT on 2012 November 18. The data were blocked into four segments of $600 \mathrm{~s}$ duration each. The actual observation time on each segment was $570 \mathrm{~s}$. The VLBI array consisted of the SRT and ground-based telescopes at Yebes, Spain (Ys); Noto, Italy (Nt); and Zelenchukskaya, Russian Federation (Zc). The diameters of these telescopes are 40,32 , and $32 \mathrm{~m}$, respectively. Over the 40-minute observation, the $(u, v)$ coordinates of the SRT-Ys baseline changed from $(1.36,2.60)$ to $(1.63,2.89)$ in units of Giga-wavelengths. The corresponding fringe spacings changed from 70 to $62 \mu$ as (corresponding to 0.049 and $0.043 \mathrm{au}$, or 7.3 and $6.4 \times$ $10^{11} \mathrm{~cm}$, respectively). The mean position angle of the space-Earth baseline was $28^{\circ}$. The $(u, v)$ coverage for the full 40 minutes is shown in Figure 2. The data were correlated using the Astro Space Center (ASC) software correlator (Likhachev et al. 2017), but only two spectral subsets of the data, which contained all known spectral components, were retained (one $8 \mathrm{MHz}$ subband in each polarization). The postcorrelation data reduction, including fringe fitting, was carried out with the PIMA calibration package (Petrov et al. 2011). Most subsequent analysis was carried out with new ad hoc software suitable for space VLBI data. The processing configuration provided 1024 channels, resulting in a channel spacing of $7.81 \mathrm{kHz}$, corresponding to $0.105 \mathrm{~km} \mathrm{~s}^{-1}$. The final processing was completed after the determination of the best orbital parameters for the SRT, which were accurate to $500 \mathrm{~m}$ in position and $0.02 \mathrm{~m} \mathrm{~s}^{-1}$ in velocity (Stepanyants et al. 2017).

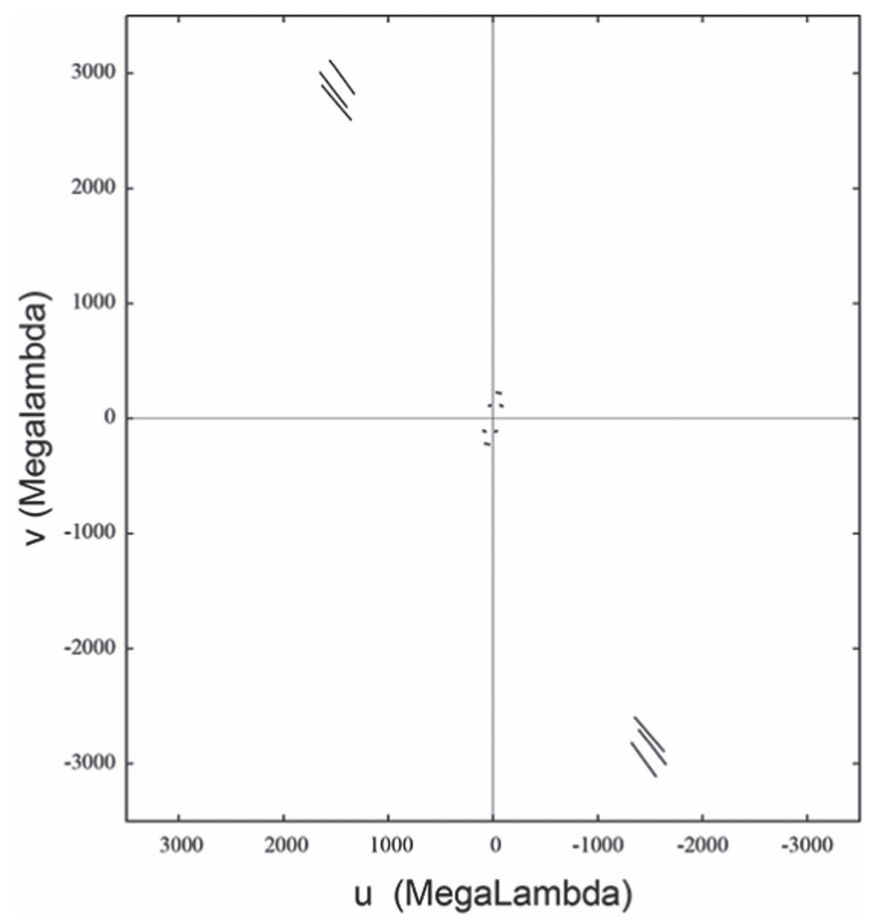

Figure 2. $(u, v)$ plane coverage of the 40-minute observation of Cepheus A on 2012 November 18 in millions of wavelengths.

\section{Results}

The total power spectrum obtained from the Yebes data is shown in Figure 3. Strong fringes were detected on all three ground baselines but only on the space baseline SRT-Ys. Weaker detections were achieved on the other space baselines but were not used in this analysis. The sensitivity of the cross power spectra was limited by the coherence time of the interferometer, which was about $100 \mathrm{~s}$. We measured the fringe rates on the three ground baselines of the spectral features at 0.6 , $-9.7,-14.8$, and -16.2 , all with respect to the feature at $-16.9 \mathrm{~km} \mathrm{~s}^{-1}$. We used the task FRMAP in the Astronomical Image Processing System (AIPS) described by Walker (1981) and Thompson et al. (2017) to find the relative feature positions from their relative fringe rates. Each relative fringe rate localized the relative position of the feature to a line in R.A.-decl. space. Although the hour angle spread provided by the 40-minute observation was small, the three ground-based baselines provide a good spread in position angle such that accurate relative coordinates were obtained with an uncertainty of \pm 0 ". 02 in each coordinate. The positions are listed in Table 1. However, it is difficult to align the masers with the continuum. We have placed the $-16.9 \mathrm{~km} \mathrm{~s}^{-1}$ feature near the center of the outflow in HW2. The absolute positions of 39 masers associated with HW2 in 1995 were reported by Torrelles et al. (1998). None of these velocity components can be reliably associated with our detections. However, most of the strong components identified in 1995 were within \pm 0 ". 3 of the center of HW2. In particular, components near our velocity commonly appear in maser complex R4 (Torrelles et al. 2011). We adopt \pm 0 ". 2 as our alignment accuracy.

Fringes on the SRT to ground baselines were detected only on the features at -16.9 and $0.6 \mathrm{~km} \mathrm{~s}^{-1}$ (the detection threshold is about $2 \mathrm{Jy}$ ). The one at $-16.9 \mathrm{~km} \mathrm{~s}^{-1}$, which is associated with HW2, had a fringe visibility amplitude of only about 0.02 . We focused our analysis on the strong isolated feature at 


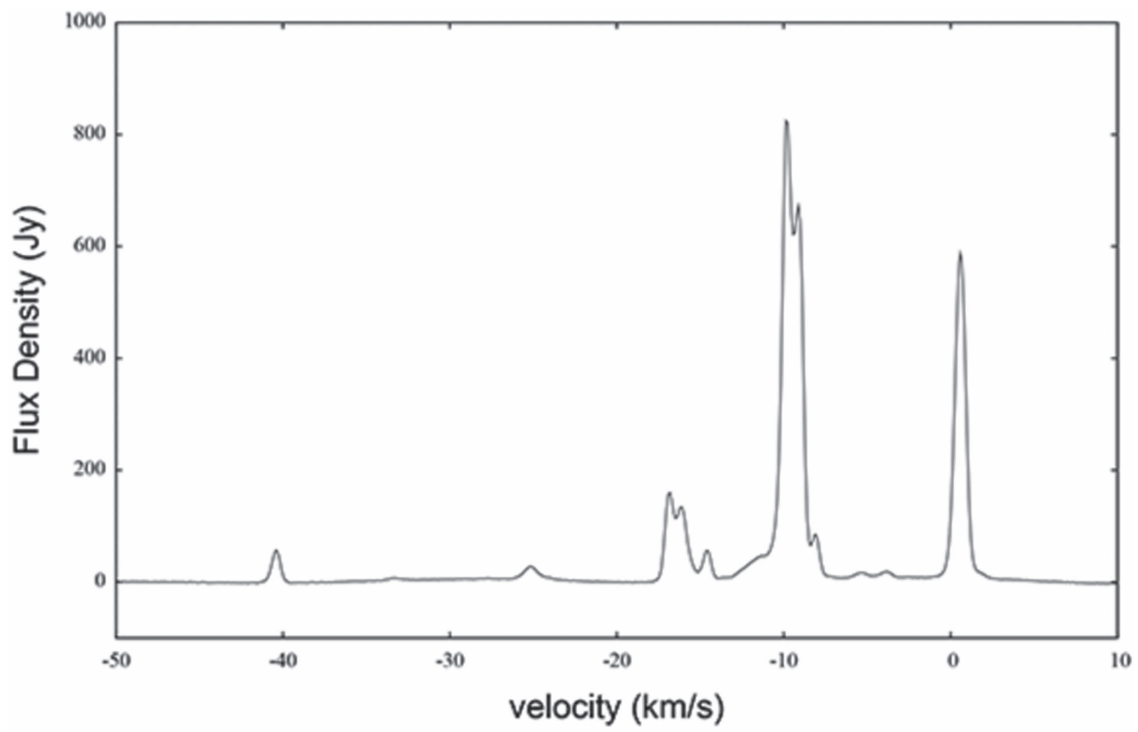

Figure 3. The total power spectrum (average of the RCP and LCP spectra) from the first $600 \mathrm{~s}$ segment of observations at the Yebes telescope. No off-source reference spectrum was available, so a polynomial baseline was fit to the signal-free parts of the spectrum and removed. The velocity is with respect to the local standard of rest (LSR). $V($ LSR $)=0 \mathrm{~km} \mathrm{~s}^{-1}$ corresponds to $V$ (heliocentric) $=-7.5 \mathrm{~km} \mathrm{~s}^{-1}$. On the 3.3 ED baselines between the SRT and ground stations, fringes were detected only on the -16.9 and $0.6 \mathrm{~km} \mathrm{~s}^{-1}$ features.

Table 1

Positions of $\mathrm{H}_{2} \mathrm{O}$ Masers in Cepheus $\mathrm{A}^{\mathrm{a}}$

\begin{tabular}{lcccl}
\hline \hline $\begin{array}{l}\text { Velocity (lsr) } \\
\left(\mathrm{km} \mathrm{s}^{-1}\right)\end{array}$ & $\begin{array}{c}\Delta \text { R.A. } \\
\left({ }^{\prime \prime}\right)\end{array}$ & $\begin{array}{c}\Delta \text { Decl. } \\
\left({ }^{\prime \prime}\right)\end{array}$ & $\begin{array}{c}\text { Flux Density } \\
(\mathrm{Jy})\end{array}$ & $\begin{array}{l}\text { Continuum } \\
\text { Association }\end{array}$ \\
\hline 0.6 & 2.29 & -3.33 & 580 & HW3diii \\
-9.7 & 1.64 & -3.10 & 800 & HW3dii \\
-14.8 & 0.05 & 0.07 & 55 & HW2 \\
-16.2 & 0.01 & 0.01 & 130 & HW2 \\
-16.9 & 0 & 0 & 152 & HW2 \\
\hline
\end{tabular}

Note.

a Relative position accuracy is \pm 0 ". 2 .

$0.6 \mathrm{~km} \mathrm{~s}^{-1}$. Routine monitoring of the spectrum at the Pushchino Observatory indicates that most features persist for about a year. In particular, the feature at $0.6 \mathrm{~km} \mathrm{~s}^{-1}$ appeared between 2012 August 30 and 2012 September 20 and disappeared between 2013 March 1 and 2013 July 18 (see Figure 4). Except for this time range, no features near this velocity were detected during the monitoring observations from 2010 October 10 to 2014 August 14. The rms noise level was typically $5 \mathrm{Jy}$. Thus, we can assign it a lifetime of $8 \pm 3$ months. The total power spectrum from our observations at Yebes is shown in Figure 5. A single Gaussian profile fit to the Stokes I spectral data $(\mathrm{RCP}+\mathrm{LCP}) / 2$ gives the parameters: amplitude $=580 \pm 3 \mathrm{Jy}$, velocity $=0.58 \pm 0.01 \mathrm{~km} \mathrm{~s}^{-1}$, and width (full width at half-maximum, $\mathrm{FWHM}$ ) $=0.672 \pm$ $0.005 \mathrm{~km} \mathrm{~s}^{-1}$. To search for the circular polarization, we calculated the Stokes V profile via the formula $V=S(\mathrm{RCP})-$ $a \times S(\mathrm{LCP})$. The parameter $a$ accounts for the small unknown gain difference between the two polarizations and was chosen to minimize the mean square deviation between $S(\mathrm{RCP})$ and $S(\mathrm{LCP})$. A longitudinal component of magnetic field in the maser medium will shift the profiles slightly in frequency. In this case, the $V$ profile has a distinctive shape

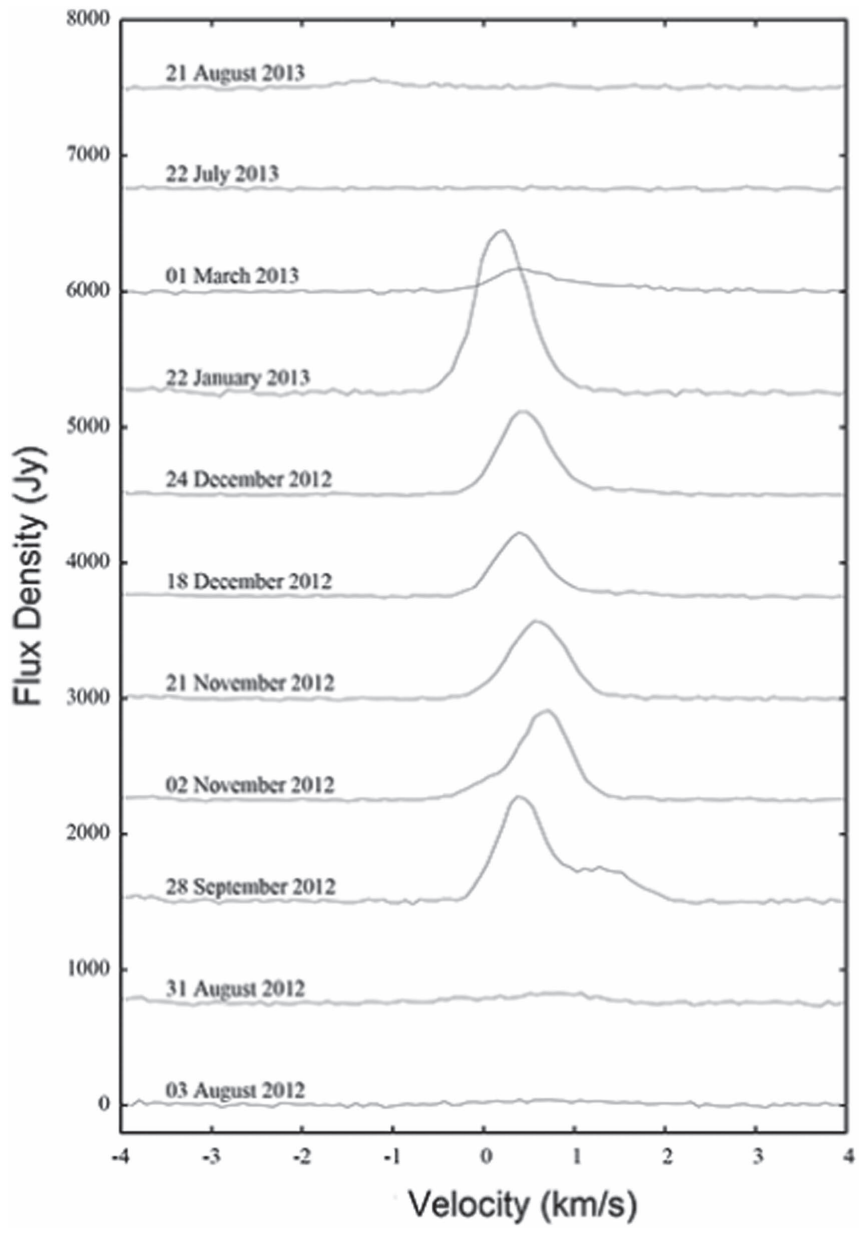

Figure 4. Spectrum of the feature near $0.6 \mathrm{~km} \mathrm{~s}^{-1}$ observed at the Pushchino Observatory from 2012 August 3 to 2013 August 21. The spectra are labeled with their dates of observation. Note the drift in the central velocity. 
proportional to the derivative of the total intensity profile. This is an anti-symmetric " $\mathrm{S}$ "-shaped curve. The magnitude of the curve, $V_{\max }$, is related to the longitudinal component of the magnetic field by the equation (Fiebig \& Güsten 1989)

$$
V_{\max } / I_{\max }=13.4 \times 10^{-6} \mathrm{~B} / \Delta v
$$

where $B$ is the line-of-sight magnetic field strength in mGauss $(\mathrm{mG})$ and $\Delta v$ is the line width in $\mathrm{km} \mathrm{s}^{-1}$. We assumed the Zeeman parameters for the strongest hyperfine component of the $22 \mathrm{GHz}$ transition with $\Delta v=0.672 \mathrm{~km} \mathrm{~s}^{-1}$. There is no hint of a Zeeman signature at the level of $1.4 \mathrm{Jy}\left(V_{\max } / I_{\max }<\right.$ $2.4 \times 10^{-3}$ ). Hence, the line-of-sight component of the magnetic field strength is less than about $120 \mathrm{mG}$. For comparison, Vlemmings et al. (2006) measured the magnetic fields in about 30 features in Cepheus A, mostly in the HW2 region, and found them typically in the range of $100-600 \mathrm{mG}$.

The vector-averaged cross power spectrum of the $0.6 \mathrm{~km} \mathrm{~s}^{-1}$ feature is shown in Figure 6. The spectrum shows two components with a sharp change in phase between them. This is a clear indication of a double source structure. We fit a double Gaussian profile to the complex cross power spectrum. The parameters of this fit are listed in Table 2. We were not able to obtain a stable three Gaussian component fit to the total power spectrum. However, we believe the total power associated with the two components cannot be significantly greater than the cross power amplitudes or they would be clearly visible in the total power spectrum (see fitted profile in Figure 5). Hence, we assign both of them visibility amplitudes of greater than 0.8 , and hence sizes of less than $15 \mu \mathrm{as}$, which leads to the estimate of the lower limits of brightness temperature in Table 2. Note that the normalized fringe visibility can be accurately determined because the total power spectrum can be measured with both the SRT and Ys telescopes. In this case, the fringe visibility is simply the cross power spectrum divided by the geometric mean of the total power spectra in raw correlator units. Individual values of system-equivalent flux densities from a priori measurements are not needed. The fraction of flux $\left(\mathrm{Jy} \mathrm{km} \mathrm{s}^{-1}\right)$ in the cross power spectrum is $0.13 \pm 0.02$ of the $0.6 \mathrm{~km} \mathrm{~s}^{-1}$ complex. This fraction is the ratio of the integrals of curve $b$ and curve a in Figure 6. To further investigate the structure of the $0.6 \mathrm{~km} \mathrm{~s}^{-1}$ component, we examined the cross power spectra on the three ground-only baselines. A careful calibration of the cross power spectra with the associated autocorrelation spectra on a minute-by-minute basis shows that the normalized fringe visibilities are $0.83,0.61$, and 0.53 for $\mathrm{Ys}-\mathrm{Nt}, \mathrm{Nt}-\mathrm{Zc}$, and Ys-Zc baselines of length 115,138 , and $228 \mathrm{M} \lambda$, respectively (see Figure 2). As mentioned above, the statistical uncertainty in these estimates is small because the system temperatures and telescope collecting areas drop out of the calculation, but the visibilities could be underestimated because of local oscillator coherence loss factors. These visibilities can be modeled approximately by a circular Gaussian disk of diameter (FWHM) of $400 \pm 15 \mu$ as and flux density of $580 \mathrm{Jy}$. We refer to this structure as a halo. Note that we could not determine the registration between the halo and the compact double structure.

The phase difference between the two components of the $0.6 \mathrm{~km} \mathrm{~s}^{-1}$ feature is about $125^{\circ}$ at the midpoint of the observations or about 0.35 of the fringe spacing, or $24 \mu \mathrm{as}$. If the features were aligned along the direction of maximum resolution at a PA of $28^{\circ}$ (see Figure 2), then they would be spaced by $24 \mu \mathrm{as}$. This is the minimum possible spacing. The actual separation and position angle can be estimated by the change in the relative phase of the features over the observations, which is $43^{\circ}$ (see Figure 7). The maximum contribution to this relative phase due to a change in instrumental delay caused by a baseline error is $\pm 2^{\circ}$ (Stepanyants et al. 2017). We thus are able to calculate a phase difference for the beginning of the observation to be $102^{\circ} \pm 10^{\circ}$ and the phase difference at the end of the observation to be $145^{\circ} \pm 10^{\circ}$. The position offset and its PA can be determined by the two $(u, v)$ plan measurements, as shown in Figure 8 . The baseline rotates by only about $3^{\circ}$, but this is sufficient to determine the offset to be $160 \pm 35 \mu$ as at a PA of $113^{\circ} \pm 5^{\circ}$. This corresponds to a projected velocity gradient of $4 \mathrm{~km} \mathrm{~s}^{-1} \mathrm{au}^{-1}$. The visibilities versus baseline length and a cartoon of the maser components are shown in Figure 9.

The question arises as to whether the size estimates of the components could be affected by interstellar scattering. The angular broadening of images due to the turbulent interstellar medium can be estimated from the NE2001 model of Cordes \& Lazio (2003). For the Galactic longitude of $109^{\circ} .8$ and latitude of $2^{\circ} .1$, the integrated effect over $700 \mathrm{pc}$ at $22.2 \mathrm{GHz}$ is $7 \mu \mathrm{as}$. Hence, scattering could have only a small effect on our measurements.

\section{Discussion}

Three properties of the $0.6 \mathrm{~km} \mathrm{~s}^{-1}$ feature clearly distinguish it from the other Cepheus A features detected in our observations: (1) the doublet structure of the feature revealing itself only at the space-ground baselines, (2) the unusual value of the radial velocity (i.e., a value not prominently represented in the cluster of masers near HW2 and HW3dii), and (3) its strong variability with nonlinear drift in velocity with time (see Figure 4). As discussed in the first subsection below, the existence of the doublet structure can have a spectroscopic explanation, but evidence also exists that the real explanation is astrophysical in nature, as discussed in the further subsections. The most likely explanation of the structure is that it results from turbulence on a variety of scales up to $400 \mu$ as. The two peaks we detected may be simply the emission peaks on the principal scale that the SRT-ground baselines are sensitive to, i.e., tens to hundreds of $\mu$ as.

In order to understand the physical nature of the $0.6 \mathrm{~km} \mathrm{~s}^{-1}$ feature, we need to determine the type of astrophysical object associated with it. Results presented in Table 1 and Figure 1 show that emission in this feature comes from the area around the compact H II region HW3diii (see Figure 1). Maps by Chibueze et al. (2012) show that the widespread maser features corresponding to the outflow in this area have proper motion velocities around $10 \mathrm{~km} \mathrm{~s}^{-1}$. The features with the other velocities, including more redshifted ones, are located in the turbulent central maser cluster. The presence of a circumstellar disk or envelope around a young stellar object (YSO) can explain the relatively high range of velocities observed. Results obtained by Chibueze et al. (2012) provide strong support for the presence of the massive YSO in the region. The very close location of the most redshifted maser feature to that of the most blueshifted one makes the disk hypothesis more likely.

Turbulent motions in the form of evolving three-dimensional vortices (eddies) are characteristic of the environment of massive YSOs. The turbulence is introduced at the largest 


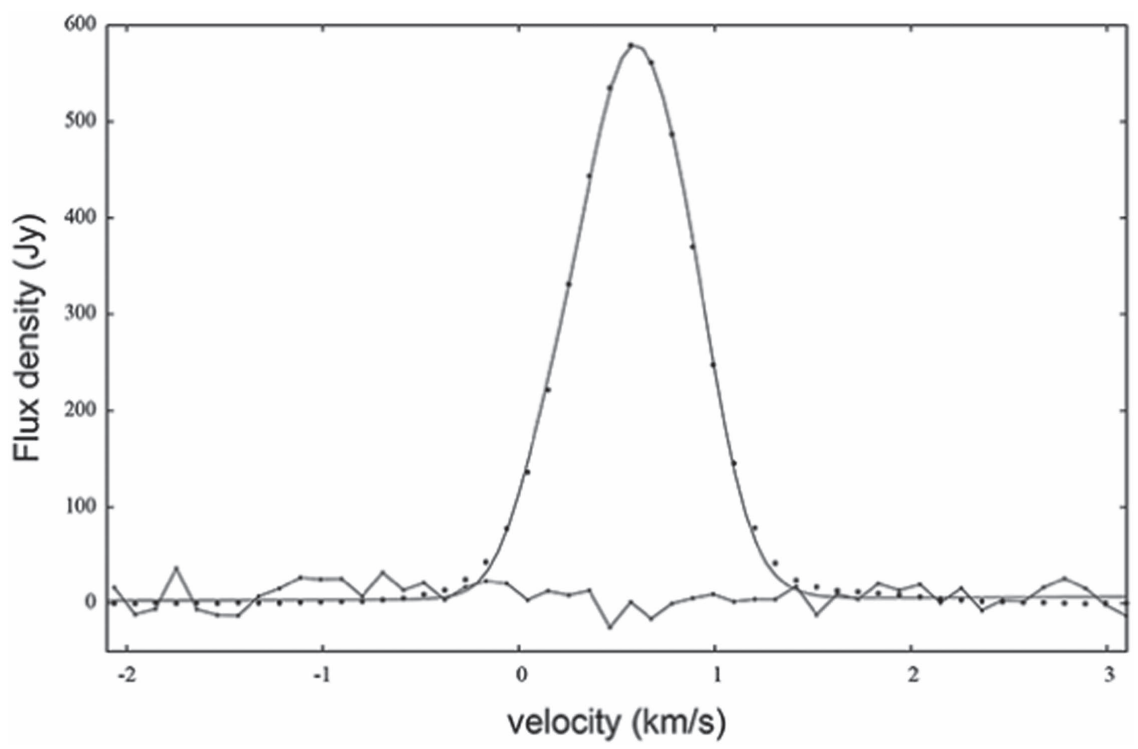

Figure 5. Close-up of the $0.6 \mathrm{~km} \mathrm{~s}^{-1}$ feature. The dots are the total power spectrum obtained from the Yebes telescope data (see full spectrum in Figure 3). The smooth line is a Gaussian profile fitted to the data. The straight line segmented curve is the difference between the RCP and LCP total power spectra after removing a gain factor. The scale of the difference spectrum has been multiplied by a factor of 20 . The absence of any significant signal indicated that the magnetic field is less than $120 \mathrm{mG}$.
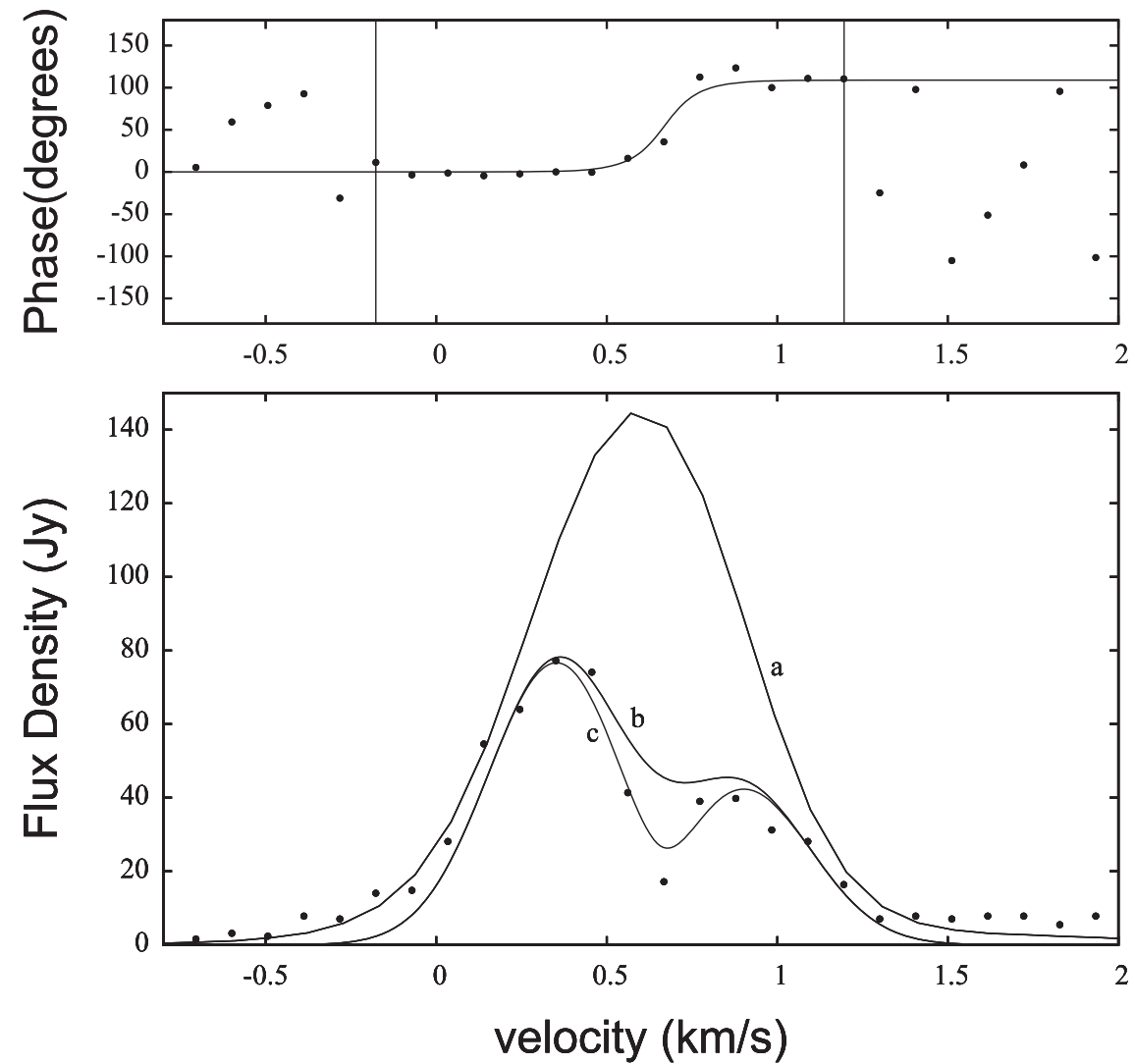

Figure 6. The cross power spectrum (dots) from the SRT-Ys baseline data for the first $600 \mathrm{~s}$ block in LCP. The visibility phase and amplitude data are shown in the top and bottom plots, respectively. A complex two-component Gaussian model was fit to this data. This model is shown by the solid line in the top plot (phase), with the velocity range marked by the vertical lines within which the signal-to-noise ratio is adequate, and by curve (c) in the bottom plot (amplitude). Curve (b) is the scalar sum of the two spectral components, and curve (a) is the total flux density reduced in scale by a factor of four for comparison.

scales determined by the boundary conditions and dissipates at the smallest scales determined by viscosity. The turbulence is generated in the circumstellar disks around massive YSOs, where it plays a decisive role in the mixing of material, momentum transfer, and other processes important for the disk structure and evolution. Excellent theoretical examples of the turbulent vortex formation in accretion disks can be found in, e.g., Meheut et al. (2010) and Kurbatov et al. (2014). Unfortunately, manifestations of a turbulent vortex in maser emission from accretion disks are much less studied 


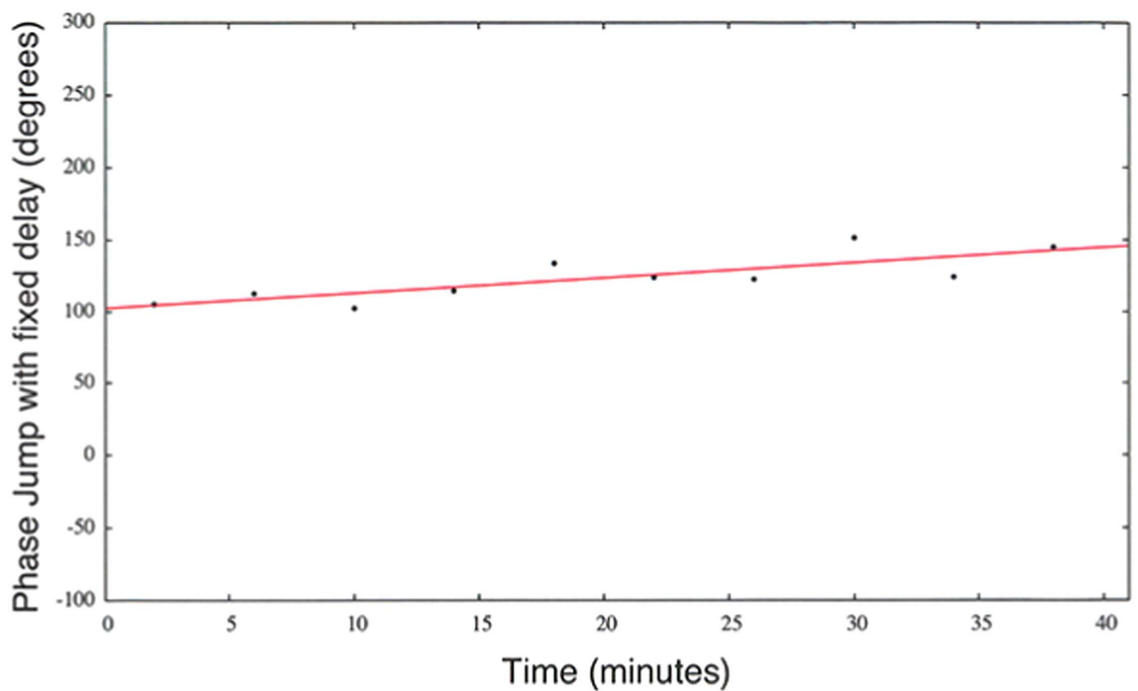

Figure 7. The relative phase between the 0.90 and $0.36 \mathrm{~km} \mathrm{~s}^{-1}$ subcomponents as a function of time during the 40 minute observation. The data have been coherently averaged to 4 minutes. The relative phase and relative phase drift over the observation of $45^{\circ}$ can be used to constrain the separation of the components. The orbit specification for RadioAstron of $0.02 \mathrm{~m} \mathrm{~s}^{-1}$ would allow a maximum of $\pm 2^{\circ}$ of the observed phase shift to be caused by the change in the baseline error. AGN observations near the time of these observations suggest that the actual error is about four times smaller.

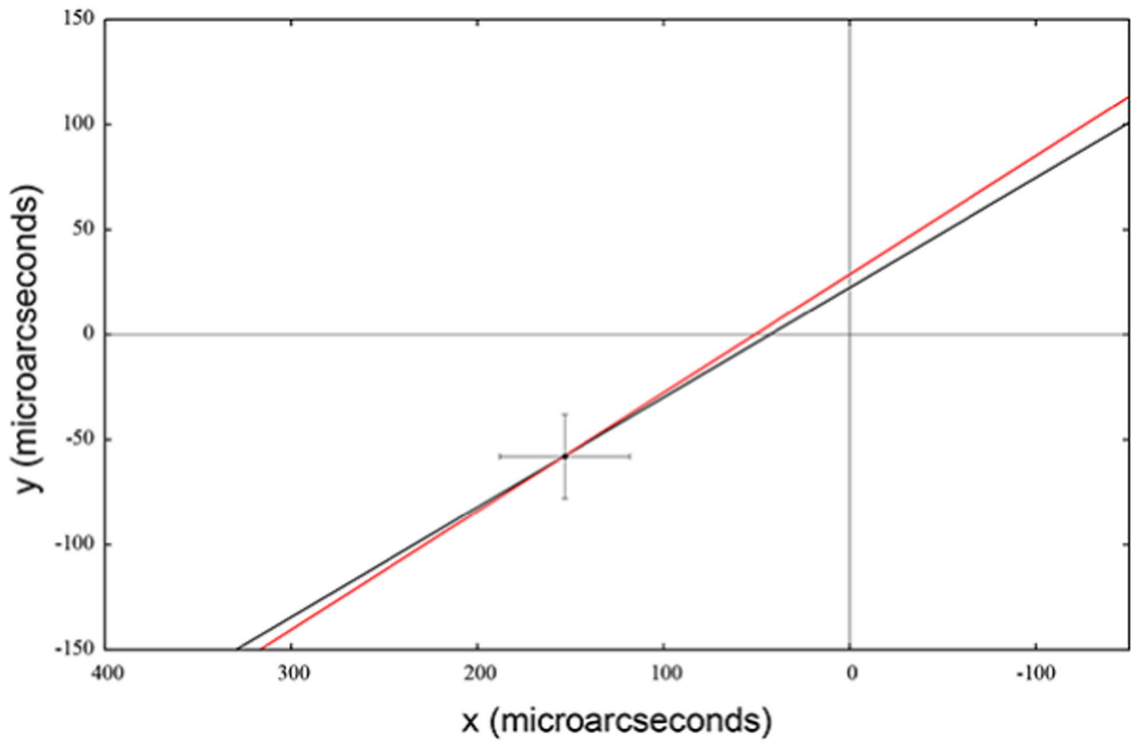

Figure 8. The offset between the 0.90 and $0.36 \mathrm{~km} \mathrm{~s}^{-1}$ subcomponents determined from relative phase measurements on the SRT-Ys baseline at 12:00 (red line) and 12:40 UT (black line). Each measurement constrains the relative position to a line in position space.

Table 2

Visibility Components of the $0.6 \mathrm{~km} \mathrm{~s}^{-1}$ Feature

\begin{tabular}{lccr}
\hline \hline $\begin{array}{l}\text { Velocity } \\
\left(\mathrm{km} \mathrm{s}^{-1}\right)\end{array}$ & $\begin{array}{c}S \\
(\mathrm{Jy})\end{array}$ & $\begin{array}{c}\Delta v \\
\left(\mathrm{~km} \mathrm{~s}^{-1}\right)\end{array}$ & \multicolumn{1}{c}{$\begin{array}{l}T_{B}{ }^{\mathrm{a}} \\
(\mathrm{K})\end{array}$} \\
\hline 0.895 & 43 & 0.47 & $1.5 \times 10^{14}$ \\
0.355 & 77 & 0.47 & $3 \times 10^{14}$ \\
\hline
\end{tabular}

Note.

${ }^{\mathrm{a}}$ Lower limit.

observationally. The main problem is the difficulty of associating maser sources with their locations in the disks. This has been addressed in only a few cases (e.g., Gallimore et al. 2003 for the R4 maser arc near Cepheus A HW2, Sanna et al. 2017 for the Cepheus A HW2 disk, and Sanna et al. 2015 for G023.01-00.41).
In contrast, in the outflows from massive YSOs, the largest and intermediate scales of turbulence are well traced by water maser observations (see the papers on W49N by Walker (1984) and Gwinn (1994a)) and more recent papers on the nearby sources Cepheus A and W75N (Uscanga et al. 2010) and W3IRS5 (Imai et al. 2002). In their consideration of the maser data on the turbulence in the flows from the massive YSOs, Strelnitski et al. (2002) proposed that "the maser hot spots originate at the sites of ultimate dissipation of highly supersonic turbulence." This assertion finds support in a wellordered spatio-kinematical pattern in the small-scale water maser features reported in Cepheus A HW2 by Uscanga et al. (2003), in W75N by Uscanga et al. (2005), and in W49N by Gwinn (1994b). Observations of Uscanga et al. (2005) suggested microstructure with a size about 1 au. This structure had a short lifetime supposedly on the order of a month. All information on the known examples of the structures with an 


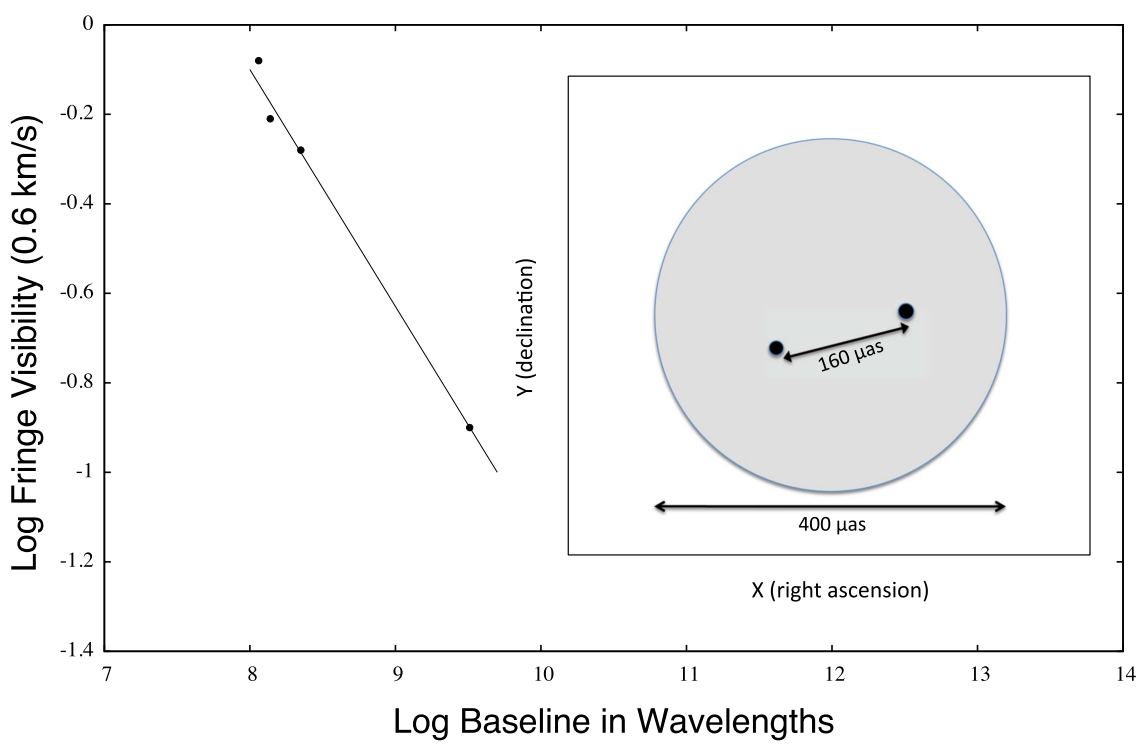

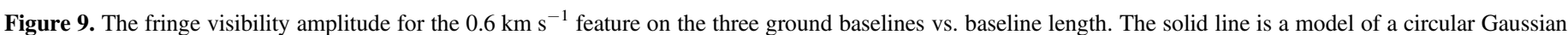

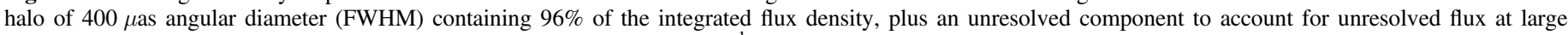

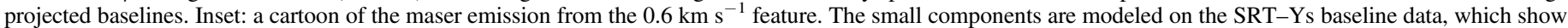

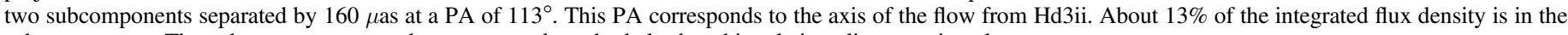
subcomponents. The subcomponents are shown centered on the halo, but this relative alignment is unknown.

"eddy-like" spatio-kinematical pattern does not contain evolutionary information and has a form of snapshots, although the other maser structures in Cepheus A HW2 show persistence on the timescales of years (Torrelles et al. 2001). Uscanga et al. (2005) speculated that these short-lived kinds of spatiokinematical microstructures are either produced by fluid instabilities within the shocked material or correspond to nearly round cloudlets (turbulent eddies?) in the ambient medium.

In the sections below, we discuss a spectroscopic origin for our observations as well as three dynamical phenomena that may explain them.

\subsection{Spectroscopic Origin: Hyperfine Splitting}

The velocity separation of $0.54 \mathrm{~km} \mathrm{~s}^{-1}$ between the components in Figure 6 is close to the velocity separations of the $\mathrm{H}_{2} \mathrm{O}$ hyperfine splittings of $0.45 \mathrm{~km} \mathrm{~s}^{-1}$ between the $F=7-6$ and $F=6-5$ transitions and $0.58 \mathrm{~km} \mathrm{~s}^{-1}$ between $F=6-5$ and $F=5-4$. However, there are several problems with this spectroscopic hypothesis. First, the components of the double-peaked spectrum in Figure 6 are not spatially coincident, so they would have to be associated with different hyperfine components. Second, the $F=7-6$ hyperfine transition has the lowest frequency (and $F=5-4$ the highest) of the three strong transitions (Kukolich 1969), while the strength order is $F=7-6, \quad F=6-5$, then $F=5-4$ (Deguchi \& Watson 1986). We would therefore expect the strongest peak at the lowest frequency (most positive Doppler shift) in our spectrum, but the opposite is seen in Figure 6. Moreover, all three of the hyperfine transitions introduced above have comparable line strengths (see Figure 1 of Deguchi \& Watson 1986), so a triplet spectrum would be expected rather than a doublet in the case of hyperfine intensity anomalies. We think this explanation is unlikely because it would require some complicated combination of hyperfine-specific pumping and/or competitive gain effects to generate the observed spectrum.

\subsection{Keplerian Rotation}

For the first dynamical interpretation, the maser hot spots (see Figure 9) might be amplifying along chords (i.e., filaments) in the plane of a Keplerian disk, orbiting a protostellar or protoplanetary object, viewed approximately edge-on. In this case, the length of the filaments responsible for the emission is displaced radially by $80 \mu$ as $\left(8.4 \times 10^{11} \mathrm{~cm}\right)$ from the center. A rotational velocity equal to $0.27 \mathrm{~km} \mathrm{~s}^{-1}$, half the velocity separation of the components, gives a central mass, $M=r v^{2} / G$, of $9.1 \times 10^{24} \mathrm{~kg}$, or approximately 1.5 Earth masses. The orbital period would be 2300 days, which is much longer than our monitoring period. A very large maser depth (negative optical depth) is possible in a disk if the number density is close to the maximum for strong collisional pumping of the $22 \mathrm{GHz}$ transition: $n=2 \times 10^{10} \mathrm{~cm}^{-3}$ at $T_{K}=750 \mathrm{~K}$ in largely dust-free gas (Gray et al. 2016). Under these conditions, a $1 \%$ inversion with an ortho- $\mathrm{H}_{2} \mathrm{O}$ abundance of $3 \times 10^{-5}$ yields a gain coefficient of $1.05 \times 10^{-10} \mathrm{~cm}^{-1}$, and therefore a maser depth would be well above the level needed to achieve saturation. Under this hypothesis, the splitting of the $0.6 \mathrm{~km} \mathrm{~s}^{-1}$ feature can be explained by rotation of the planetary object around the massive YSO in the region.

\subsection{A Pair of Approximately Spherical Clouds}

The second dynamical interpretation is that the $0.6 \mathrm{~km} \mathrm{~s}^{-1}$ maser emission results from the partial overlap, along the line of sight, of a pair of approximately spherical clouds. This alignment could be random, although it is much more likely that the objects are related. The clouds may have a very large relative velocity, provided that the dominant component lies in the plane of the sky. The relative velocity along the line of sight needs to be comparable to the Doppler-broadened line width, which is the same in both clouds. If this is the case, radiation at some frequencies will be amplified along the line of sight through a medium that combines material from both clouds. If the centers of the clouds pass close to each other along the line of sight, the likely result is a maser flare; see, for example, 
Lekht et al. (2009). The object we observe in Cepheus A would, in this scenario, be either a pre- or post-flare object, depending on whether the clouds are approaching, or separating from, their minimum line-of-sight separation. Multi-epoch observations would be necessary to test this model via proper motion analysis. At any frequency in the spectrum of the overlapping clouds, a ray amplifying through the overlapping region will pass through an optical depth $\tau_{1}$ of material from the first cloud and $\tau_{2}$ from the second cloud, with a resulting spectrum as shown, for example, in Figure 9 of Lekht et al. (2009). We note that the differently shifted central response frequencies of the two clouds imply that the greatest optical depth, at a particular frequency, does not in general correspond to the greatest combined path length through the clouds, even if the lengths are otherwise identical. Comparison with our Figure 6, lower panel, suggests that our pair of clouds would be somewhat less overlapped than the Lekht et al. examples. Also, the model of only a pair of approximately spherical clouds does not naturally explain the variability pattern of the Pushchino monitoring. A more realistic model may involve nonspherical clouds or more overlapping clouds. In fact, this brings us close to the turbulence hypothesis discussed in the next section but without a pronounced turbulent vortex.

\subsection{Structures in a Turbulent Flow}

In the third dynamical model, we consider the case of turbulent vortices shed from the dense gas formation. Vortex formation, shedding, and evolution in the flow over a dense obstacle are widely discussed in the literature, (e.g., Loytsansky 1970; Blevins 1990; Lienhard \& Lienhard 2011). The turbulent motions have different regimes that are described by a set of dimensionless numbers (criteria). The corresponding regime of an unestablished flow is usually characterized by the Strouhal number, St. Expressed in observational parameters, it is equal to the ratio of characteristic scale, $R$, to the product of a characteristic speed, $v$, and characteristic time, $\tau$, i.e., $S t=$ $R /(\tau v)$. (Note: the Strouhal number is often defined as $S t^{-1}$.) This number represents the ratio of the local velocity derivative to the convective derivative in the Navier-Stokes equation. Thus, this number describes the ability of the flow to form persistent turbulent vortices. The basic property of this criterion is that the Strouhal number $S t$ has values from about 0.2 to about 0.3 for a wide range of Reynolds numbers, $R e$ (see mentioned textbooks, report by Roshko 1954, and relatively recent experimental study by Shi et al. 2011 and theoretical study by Ponta \& Aref 2004). In order to facilitate discussion of our observations, we write $S t$ as

$$
S t=57 R_{\mathrm{au}} /\left(\tau_{m} v_{\mathrm{kms}}\right),
$$

where $\tau_{m}$ is the time in months, $v_{\mathrm{kms}}$ is the velocity in $\mathrm{km} \mathrm{s}^{-1}$, and $R_{\mathrm{au}}$ is the spatial scale in astronomical units.

Pushchino monitoring shows that the $0.6 \mathrm{~km} \mathrm{~s}^{-1}$ feature in Cepheus $\mathrm{A}$ at the time of our observations experienced a rather strong flare, which is not likely to be periodic. Figure 4 shows that the flare lasted for about eight months and had two peaks at slightly different velocities. These peaks may correspond either to the full cycle of a single-vortex rotation or to formation of two different vortices.

Under the single-vortex hypothesis, the two maser spots correspond to two edges of the vortex. To estimate the Strouhal number, we adopt $\tau_{m}=16 \pm 6$, twice the lifetime of the
$0.6 \mathrm{~km} \mathrm{~s}^{-1}$ maser flare. We doubled the lifetime because (1) the full cycle of rotation implies that emission peak returns to the same velocity and (2) the arc in the position-velocity dependence of the $0.6 \mathrm{~km} \mathrm{~s}^{-1}$ feature (see Figure 4) suggests that it lasts for about half of a full cycle. Further, we assume that the component velocity difference $0.54 \mathrm{~km} \mathrm{~s}^{-1}$ corresponds to a velocity difference of the edges of the vortex. Under this assumption, the characteristic velocity should be half of this value, i.e., $v_{\mathrm{kms}}=0.27$, and the measured value of the separation of the two components $R_{\mathrm{au}}=0.11(160 \mu \mathrm{as})$ should be about the vortex diameter. The resulting Strouhal number is $S t \sim 1.5$, which is out of the normal range even for the cases of very high Reynolds numbers (Green 1995; Schewe 1983). Hence, we consider the hypothesis of the single turbulent vortex to be unlikely.

In the two-vortex interpretation, each maser spot represents a vortex that forms in the wake of an obstacle in an outflow (von Kármán street vortices). In our case, the line between the maser spots corresponds well to the axis of the outflow observed by Chibueze et al. (2012), and we consider this outflow as the progenitor of vortex formation. It is possible that the obstacle is associated with HW3diii. Subsequent vortices in the street rotate in the opposite sense. The density of the vortices decreases with the distance from the obstacle, so the dense gas responsible for the bright maser emission is present only in close proximity to the obstacle, so we observe only the first two. Vortex shedding has the following phases: (1) formation of one vortex with a component of velocity toward the flow axis on one side of the obstacle, (2) formation of another vortex with a component of its velocity toward the flow axis on the other side of the obstacle (at which stage we observe two dense vortices moving toward the flow axis from opposite sides), and (3) the vortices approach the flow axis and start moving along the flow (in the meantime, a new vortex starts forming). When the obstacle is not symmetric, the vortices formed on one side of the obstacle can be denser, bigger, and, hence, brighter in maser emission. This model is consistent with the Pushchino monitoring results in Figure 4 under the hypothesis that strong flares correspond to vortex formation, and we observe these structures moving along the flow axis. We should then observe two scales: the larger scale corresponds to vortex separation, or obstacle size (about 0.11 au in our case), and the smaller scale, to two vortices with opposing rotation that manifest themselves at the highest angular resolution (our observed unresolved structures). Turbulence would therefore dissipate on scales much smaller than $0.11 \mathrm{au}$ in this region. Temporally, the period of the vortex shedding will correspond to half of the time difference between the strong flares, so about two months for the data in Figure 4; its characteristic velocity is about $10 \mathrm{~km} \mathrm{~s}^{-1}$, from typical proper motions measured by Chibueze et al. (2012), and the characteristic size is about 0.11 au. These parameters give $S t=0.3$, a plausible value for a turbulent flow in the interstellar medium. The hypothesis of a pair of turbulent vortices formed by an obstacle in the flow is therefore consistent with both the RadioAstron and Pushchino data from Figure 4.

\section{Conclusion}

We have investigated the structure of a single maser "spot" in the Cepheus A region. We found that the maser spot had a total extent of about $400 \mu \mathrm{as}$. It is threaded by a magnetic field of less than $120 \mathrm{mG}$. The substructure is undoubtedly complex, 
but it includes two prominent structures separated by $160 \mu$ as, which contain about $13 \%$ of the flux. The high contrast suggests that they may be unsaturated lines of sight. They may correspond to a pair of turbulent eddies shed by an obstacle in a flow, i.e., a Kármán vortex street with Strouhal number of about 0.3 , to objects bound in orbit by a planetary size mass, or to individual filaments or overlapping spherical clouds.

We note that the current study lacks information on the intermediate baselines, which are essential for accurate image recovery. Involvement of the High-Sensitivity Array or full VLBA in observations of Cepheus A in combination with RadioAstron would help to elucidate whether we have resolved the smallest scale of the turbulence, which is a basic parameter for understanding the evolution and structure of the interstellar medium of star-forming regions. Observations of flares should be conducted at intervals of a few months to determine their temporal and spatial characteristics.

We thank Vladimir Kostenko, Vyacheslav Avdeev, and Pyotr Voitsik for help with correlation and calibration issues and Mark Reid for helpful suggestions on the manuscript. The RadioAstron project is led by the Astro Space Center of the Lebedev Physical Institute of the Russian Academy of Sciences and the Lavochkin Scientific and Production Association under a contract with the Russian Federal Space Agency, in collaboration with partner organizations in Russia and other countries. Partly based on observations performed with radio telescopes of IAA RAS (Institute of Applied Astronomy of the Russian Academy of Sciences). Partly based on observations with the Noto telescope operated by the Istituto di Radioastronomia di Bologna. Partly based on observations with the $40 \mathrm{~m}$ radio telescope of the Yebes Observatory of the IGN (Instituto Geográfica Nacional, Spain). Technical support was received from the National Space Facilities Control and Test Center. Results of optical position measurements of the Spektr-R spacecraft (the platform for the RadioAstron Space Radio Telescope) by the global MASTER Robotic Net, ISON collaboration, and the Kourovka Astronomical Observatory of the UrFU (Ural Federal University) were used for spacecraft orbit determination in addition to mission facilities. This work was supported in part by the Ministry of Education and Science (the basic part of the State assignment, RK no. AAAA-A17-117030310283-7) and by the Act no. 211 of the Government of the Russian Federation, agreement 02. A03.21.0006.

Facilities: RadioAstron Space Radio Telescope (Spektr-R), Yebes Radio Observatory (Instituto Geográfica Nacional, Spain), Noto Radio Observatory (Istituto di Radioastonomia di Bologna), and Zelenchukskaya Radio Observatory.

Software: ASC software correlator (Likhachev et al. 2017), PIMA (Petrov et al. 2011), AIPS (van Moorsel et al. 1996).

\section{References}

Baan, W. A., Alakoz, A., An, T., et al. 2018, in IAU Symp. 336, Astrophysical Masers: Unlocking the Mysteries of the Universe, ed. A. Tarchi, M. J. Reid, \& P. Castangia (Cambridge: Cambridge Univ. Press), in press (arXiv:1801.08796)

Blevins, R. D. 1990, Flow-Induced Vibration (2nd ed.; New York: Van Nostrand Reinhold)
Chibueze, J. O., Imai, H., Tafoya, D., et al. 2012, ApJ, 748, 146

Cordes, J. M., \& Lazio, T. J. W. 2003, arXiv:astro-ph/0301598

Curiel, S., Ho, P. T. P., Patel, N. A., et al. 2006, ApJ, 638, 878

Deguchi, S., \& Watson, W. D. 1986, ApJ, 302, 750

Dzib, S., Loinard, L., Rodríguez, L. F., Mioduszewski, A. J., \& Torres, R. M. 2011, ApJ, 733, 71

Fiebig, D., \& Güsten, R. 1989, A\&A, 214, 333

Gallimore, J. F., Cool, R. J., Thornley, M. D., \& McMullin, J. 2003, ApJ, 586,306

Gray, M. 2012, Maser Sources in Astrophysics (Cambridge: Cambridge Univ. Press)

Gray, M. D., Baudry, A., Richards, A. M. S., et al. 2016, MNRAS, 456, 374 Green, S. I. (ed.) 1995, Fluid Vortices: Fluid Mechanics and Its Applications (Dordrecht: Kluwer)

Gwinn, C. R. 1994a, ApJ, 429, 241

Gwinn, C. R. 1994b, ApJ, 429, 253

Hughes, V. A., Cohen, R. J., \& Garrington, S. 1995, MNRAS, 272, 469

Hughes, V. A., \& Wouterloot, J. G. A. 1984, ApJ, 276, 204

Imai, H., Deguchi, S., \& Sasao, T. 2002, ApJ, 567, 971

Kardashev, N. S., Khartov, V. V., Abramov, V. V., et al. 2013, ARep, 57, 153

Kobayashi, H., Shimoikura, T., Omodaka, T., \& Diamond, P. J. 2000, in Astrophysical Phenomena Revealed by Space VLBI, ed. H. Hirabayashi, P. G. Edwards, \& D. W. Murphy (Sagamihara: Institute of Space and Astronautical Space), 109

Kukolich, S. G. 1969, JChPh, 50, 3751

Kurbatov, E. P., Bisikalo, D. V., \& Kaygorodov, P. V. 2014, PhyU, 57, 787

Lekht, E. E., Silant'ev, N. A., \& Alekseeva, G. A. 2009, ARep, 53, 813

Lienhard, J. H., IV, \& Lienhard, J. H., V 2011, A Heat Transfer Textbook (4th ed.; Cambridge, MA: Phlogiston Press), http://web.mit.edu/lienhard/ www/ahtt.html

Likhachev, S. F., Kostenko, V. I., Girin, I. A., et al. 2017, JAstrInst, 6, 1750004

Loytsansky, L. G. 1970, in Fluid and Gas Mechanics (Moscow: Nauka), 904

Meheut, H., Casse, F., Varniere, P., et al. 2010, A\&A, 516, A31

Moscadelli, L., Reid, M. J., Menten, K. M., et al. 2009, ApJ, 693, 406

Patel, N. A., Curiel, S., Sridharan, T. K., et al. 2005, Natur, 437, 109

Petrov, L., Kovalev, Y. Y., Fomalont, E. B., \& Gordon, D. 2011, AJ, 142, 35

Ponta, F. L., \& Aref, H. 2004, PhRvL, 93, 084501

RadioAstron User Handbook 2018, version 2.91, http://www.asc.rssi.ru/ radioastron/documents/rauh/en/rauh.pdf

Roshko, A. 1954, Technical Report 1191 (Washington, DC: National Advisory Committe for Aeronautics), http://authors.library.caltech.edu/428/1/ ROSnacarpt1191.pdf

Sanna, A., Moscadelli, L., Surcis, G., et al. 2017, A\&A, 603, A94

Sanna, A., Surcis, G., Moscadelli, L., et al. 2015, A\&A, 583, L3

Schewe, G. 1983, JFM, 133, 265

Shakhvorostova, N. N., Alakoz, A. V., \& Sobolev, A. M. 2018, in IAU Symp. 336, Astrophysical Masers: Unlocking the Mysteries of the Universe, ed. A. Tarchi, M. J. Reid, \& P. Castangia (Cambridge: Cambridge Univ. Press) in press (arXiv:1802.05120)

Shi, L., Yu, Z., \& Jaworski, A. J. 2011, EJMF, 30, 206

Sobolev, A. M., Shakhvorostova, N. N., Alakoz, A. V., et al. 2018, in IAU Symp. 336, Astrophysical Masers: Unlocking the Mysteries of the Universe, ed. A. Tarchi, M. J Reid, \& P. Castangia (Cambridge: Cambridge Univ. Press), in press (arXiv:1802.05133)

Stepanyants, V., Zaslavsky, G., Zakhvatkin, M., et al. 2017, presentation at the RadioAstron Int. Science Council Meeting (Moscow, Russia)

Strelnitski, V., Alexander, J., Gezari, S., et al. 2002, ApJ, 581, 1180

Thompson, A. R., Moran, J. M., \& Swenson, G. W., Jr. 2017, in Interferometry and Synthesis in Radio Astronomy (3rd ed.; Switzerland: Springer), 631

Torrelles, J. M., Gómez, J. F., Garay, G., et al. 1998, ApJ, 509, 262

Torrelles, J. M., Patel, N. A., Curiel, S., et al. 2011, MNRAS, 410, 627

Torrelles, J. M., Patel, N. A., Gómez, J. F., et al. 2001, ApJ, 560, 853

Uscanga, L., Cantó, J., Curiel, S., et al. 2003, RMxAC, 15, 147

Uscanga, L., Cantó, J., Curiel, S., et al. 2005, ApJ, 634, 468

Uscanga, L., Cantó, J., Gómez, J. F., et al. 2010, ApJ, 715, 132

van Moorsel, G., Kemball, A., \& Greisen, E. 1996, adass V, 101, 37

Vlemmings, W. H. T., Diamond, P. J., van Langevelde, H. J., \& Torrelles, J. M. 2006, A\&A, 448, 597

Walker, R. C. 1981, AJ, 86, 1323

Walker, R. C. 1984, ApJ, 280, 618 